\title{
DISCLAIMER
}

This document was prepared as an account of work sponsored by an agency of the United States Government. Neither the United States Government nor the University of California nor any of their employees, makes any warranty, express or implied, or assumes any legal liability or responsibility for the accuracy, completeness, or usefulness of any information, apparatus, product, or process disclosed, or represents that its use would not infringe privately owned rights. Reference herein to any specific commercial product, process, or service by trade name, trademark, manufacturer, or otherwise, does not necessarily constitute or imply its endorsement, recommendation, or favoring by the United States Government or the University of California. The views and opinions of authors expressed herein do not necessarily state or reflect those of the United States Government or the University of California, and shall not be used for advertising or product endorsement purposes.

This work was performed under the auspices of the U. S. Department of Energy by the University of California, Lawrence Livermore National Laboratory under Contract No. W-7405-Eng-48.

This report has been reproduced

directly from the best available copy.

Available to DOE and DOE contractors from the Office of Scientific and Technical Information

P.O. Box 62, Oak Ridge, TN 37831

Prices available from (423) 576-8401

http://apollo.osti.gov/bridge/

Available to the public from the

National Technical Information Service

U.S. Department of Commerce

5285 Port Royal Rd.,

Springfield, VA 22161

http://www.ntis.gov/

OR

Lawrence Livermore National Laboratory

Technical Information Department's Digital Library

http://www.llnl.gov/tid/Library.html 


\section{A Report of the Joint Development of a Prototype Communications Link to Share Nuclear Accident Dispersion and Dose Assessment Modeling Products Between JAERI/WSPEEDI and LLNL/NARAC}

\author{
Thomas J. Sullivan \\ Richard D. Belles \\ James S. Ellis \\ Lawrence Livermore National Laboratory \\ Livermore, California USA 94551
}

\author{
Masamichi Chino \\ Haruyasu Nagai \\ Japan Atomic Energy Research Institute \\ Tokai-mura, Ibaraki-ken Japan
}

\section{INTRODUCTION}

In June of 1997, under an umbrella Memorandum of Understanding between the Japan Atomic Energy Research Institute (JAERI) and the U.S. Department of Energy (US/DOE) concerning matters of nuclear research and development, a Specific Memorandum of Agreement (SMA) entitled "A Collaborative Programme of Development of a Prototype Communication Link to Share Atmospheric Dispersion and Dose Assessment Modelling Products" was signed. This SMA formalized an informal collaborative exchange between the DOE's Lawrence Livermore National Laboratory (LLNL) Atmospheric Release Advisory Capability (ARAC) center and the Japan Atomic Energy Research Institute (JAERI) Worldwide System for Prediction of Environmental Emergency Dose Information (WSPEEDI). The intended objective of this agreement was to explore various modes of information exchange, beyond facsimile transmission, which could provide for the quick exchange of information between two major nuclear emergency dose assessment and prediction national centers to provide consistency checks and data exchange before public release of their calculations. The extreme sensitivity of the general public to any nuclear accident information has been a strong motivation

to seek peer preview prior to public release. Other intended objectives of this work are the development of an affordable/accessible system for distribution of prediction results to other countries having no prediction capabilities and utilization of the link for collaboration studies.

To fulfill the objectives of this project JAERI and LLNL scientists determined to assess the evolving Internet and rapidly emerging communications application software. Our timing was a little early in 1997-1998 but nonetheless a few candidate software packages were found, evaluated and a selection was made for initial test and evaluation. Subsequently several new candidate software packages have arrived, albeit with limitations. This report outlines the ARAC 
and JAERI emergency response assessment systems, describes the prototype communications protocol system established and the tools evaluated in that process. Three real-time applications of the information exchange protocol and lessons learned are discussed and then some conclusions and future plans are presented.

\section{LLNL/ARAC AND JAERI/WSPEEDI}

\section{LLNLARAC}

The Atmospheric Release Advisory Capability, better known as ARAC, is a hybrid system of models, computers, databases, communications and highly skilled staff dedicated to emergency consequence analysis and prediction of atmospheric hazardous material releases (1). The ARAC system is located at and operated by Lawrence Livermore National Laboratory (in Livermore, California, USA). It's development and operational support for the U.S. government have been funded by the U.S. Departments of Energy and Defense for the purpose of providing real-time, down-wind consequence assessments for emergency responders and managers for radiological and other hazardous releases. This service is available for both fixed facilities and any location in the world whenever and wherever the U.S. government has interest or concern.

Over the past 27 years ARAC has provided consequence assessments for more than 200 potential and actual hazardous releases. This capability has been applied to diverse real-world releases such as the 1978 re-entry of the COSMOS 954 nuclear powered satellite over Canada, the Three Mile Island and Chernobyl nuclear power plant accidents, the Tomsk nuclear facility accident in Russia, two radiological accidents at Tokai (Japan) the Algeciras (Spain) melt of a radiotherapy source, and several non-radiological events such as chemical releases, toxic fires including the Kuwait oil fires, and even volcanic ash emissions.

Since 1975 the U.S. Department of Energy has invested in the development and advancement of technical capabilities of the Atmospheric Release Advisory Capability (ARAC) (2). The motivation for this investment is the need to have the best possible accident emergency assessments of (potential) exposure and rapid graphical depictions available to all responding levels of authority in order to effectively contribute to all phases of consequence management.

ARAC technology had evolved over the first 20 years through two generations of system architectures and with only incremental improvements in the diagnostic wind field and 
dispersion models. In 1994, a third and much larger modernisation programme was begun to both advance the system architecture and to upgrade the physics structure in the diagnostic wind and dispersion models, and to integrate mesoscale prognostic meteorological models into the capability. The existing architecture, called ARAC-2 $\left(2^{\text {nd }}\right.$ generation) was a 15-year old proprietary technology that became more difficult to maintain as a viable capability to address fully the problems of today. The new ARAC-3 ( $3^{\text {rd }}$ generation) capability provides portability to the system so that it is computer-platform-independent, a necessity in today's dynamically changing environment of computer hardware and operating system technology, and incorporates state of the art models.

The ARAC Program emergency operation center and staff moved into a newly completed building in January 1996. This facility, called the National Atmospheric Release Advisory Center (NARAC), was built by the Department of Energy to house ARAC in a "purpose designed" facility with substantial redundancy in utilities and adequate room for operations and growth.

During the past 21 years that ARAC has been an operational service, tremendous advances have occurred in communications, computers, databases, atmospheric models and software technology. The evolution of computer operating systems from "proprietary" to "open" architectures, the exceptionally cost competitive performance of new computers, the availability and transportability of system services software and the enormous data resources available via the Internet have provided the opportunity for a quantum improvement in the quality, timeliness and temporal extent of assessment forecasts for emergency management officials.

The historic ARAC diagnostic wind flow and dispersion models employed a uniform grid resolution in both the horizontal and vertical dimensions, and used a "block" representation of the terrain. This "stair-step" representation restricted the models to a rather crude description of the terrain of interest. In the new system, the block terrain representation is replaced with a continuous representation of the terrain. The grid structure of the new system is based on a terrain-following coordinate system and contains the option of varying the grid resolution in both the horizontal and vertical dimensions. This representation not only creates more realism in the models, but eliminates numerical problems associated with wind flow and dispersion in artificially stepped terrain, allows for finer resolution gridding to be used when required, and is compatible with the continuous terrain representation in the companion atmospheric prognostic models. 
As part of the ARAC modernization effort, a new Lagrangian particle transport and diffusion model, LODI (3), was developed and implemented. The previous code (ADPIC) used a hybrid Gaussian and gradient diffusion scheme. For point sources and sub-grid scale area sources, Gaussian diffusion was used until the size of the initial cloud increased by dispersion to fill one or more grid cells, and then gradient diffusion was applied. The new model is a Monte Carlo approach to the diffusion process using the random displacement method (RDM) (4). The RDM diffusion is fully grid cell independent and eliminates grid resolution difficulties associated with typical gradient diffusion approaches when point sources are modeled.

A rectangular nested grid structure of up to four inner nests was used previously to sample the cloud of Lagrangian marker particles close in to the source. The new LODI model allows a high resolution sampling near the source with an almost arbitrary choice of ever increasing cell sizes as one moves away from the source location. This eliminates discontinuities in the plume edges that often occur with the nested grid sampling because of the discrete boundaries of each nest.

A "hybrid particle" version of LODI has been implemented. Earlier versions of the ADPIC model were limited to dispersing up to nine radionuclides in any one model run. The hybrid particle version of LODI disperses a complete and unlimited mix of radionuclides, while not only allowing the radioactive decay of each radionuclide in the mix during dispersion and deposition, but spawning all the daughter products and then adds these to the mix during the model run. This new capability greatly enhances ARAC's flexibility in dealing with the mix of radionuclides that may be released from a nuclear power plant or fuel processing accident.

The new diagnostic wind field model $\operatorname{ADAPT}(5,6)$ has an improved capability to assimilate meteorological data that is obtained from multiple sources (i.e. surface, rawindsonde, multi-level meteorological tower, SODAR, LIDAR, microwave profiler, and gridded). As with LODI, the ADAPT model uses a terrain-following coordinate system which provides continuous representation of the terrain. The variable resolution of the new grid has the capability to increase the resolution of the wind field over meteorological mesonets that are imbedded in regions of widely spaced meteorological observations. It allows ARAC to incorporate multi-level tower, SODAR, LIDAR, and microwave profiler data in the vertical dimension while modeling a deep lower atmosphere. This new model also improves the capability for maintaining the integrity of the input vector winds (speed and direction) via a new, finite-element-based, massconsistent adjustment process. 
As part of the modernization effort, ARAC adapted and integrated the U.S. Navy's operational prognostic model known as the Coupled Ocean/Atmosphere Mesoscale Prediction System (COAMPS) into ARAC's operational architecture (7). COAMPS is a sophisticated, computationally intensive non-hydrostatic full atmospheric physics model designed to span the resolution scales of 1-20 kilometers. The near-term objective for these mesoscale prognostic models is to rapidly generate detailed regional atmospheric flow fields in space and time anywhere worldwide for emergency response assessments. Longer term objectives are to generate detailed cloud and precipitation fields that may impact the transport and deposition of hazardous materials.

Integration of the prognostic model into an operational emergency system poses a formidable challenge with respect to generating advisory products in a real-time mode. The reason is that forecast models typically require a significant amount of computational time relative to diagnostic models. Our strategy for deploying prognostic models in a real-time mode is to generate $48 \mathrm{hr}$ limited area forecasts at $15 \mathrm{~km}$ or less resolution, once daily, for two regions encompassing most of the sites supported by ARAC (e.g., domains centered over the East and West Coasts of the U.S.). The meteorological forecast fields are updated once every 24 hours based on the most current imported global analysis and are then used as gridded data for the ADAPT and LODI models. If an emergency occurs outside of the forecasted domains, initial response is rendered by the diagnostic models using local observational data and gridded data from available global forecasts, such as from NOGAPS or AVN forecasts (both containing about $1^{\circ}$ grid resolution information). The COAMPS forecast domain is then relocated to the region of interest and the prognostic model will begin computations, at finer resolutions, with the most recent global analysis and forecasts as initial and boundary conditions. The goal is to generate an initial prognostic emergency response calculation of a $24 \mathrm{hr}$ forecast within 4 hours of the start of the event. ARAC computer scientists have reduced the computational time via implementation of multiprocessing techniques in conjunction with coding modifications to increase the computational efficiency of the model (8).

In the new ARAC system, the dispersion model is driven directly from the forecast grids or indirectly by passing the forecast grids to the diagnostic wind field model. The diagnostic model ingests both the regional gridded data for COAMPS as well as the local on-site observational data and, with appropriate blending, generates high-resolution wind fields that are properly mass-adjusted relative to the continuous terrain. 
The new ARAC-3 computer software system has been developed to provide many improved emergency response capabilities to the ARAC Program. It is a complex integrated system using many new computer technologies and consisting of a large number of components that must interact in a coherent fashion. While the atmospheric dispersion models are the backbone of ARAC, the software system and environment that surrounds these models is critical to ARAC's ability to respond effectively and efficiently to emergencies and exercises. The entire concept and structure of the ARAC-2 supporting software that included model parameters, the model execution framework, the automation processes, the data acquisition and communication processes, the database interfaces and file structures, and the visualization tools have been modernized under the new ARAC-3 System. The computer language of choice for the models is FORTRAN 90 while the choice for the supporting software is $\mathrm{C}++$ and Java. The new system employs the widely-used UNIX operating system to allow for transportability on UNIX-based platforms and the netCDF data format for portability and standardized data protocol.

A clear priority for an emergency response system is ease of organizing the specification of an assessment (model) sequence and automated execution of necessary functionality. The ARAC system manages, displays, and analyzes the vast amounts of data required as well as manages the results generated by the models. The manner in which event information is stored forms a central component of the ARAC-3 System. This event information describes a particular release or set of releases, the metdata (meteorological data) related to that situation, the land surface surrounding the incident, model input parameters, etc. It is a key functionality of the system to unify all the interdependent sets of information providing quick and easy access to and control of all critical information (accident time, location, source description(s), terrain information, etc.).

A major function of the system is the automated creation of a set of consistent, intelligent parameters for the atmospheric models. These parameters describe the source location, type of release, the geometry of the release, the types of plots that will be generated, the dose pathways, final health effects units, emergency action concentration levels, etc.; some are meteorologically dependent, others are agency dependent. These parameters may be edited using a library of possible algorithms for the calculation of initial values. The execution and control of the atmospheric models is performed by an extensible model execution framework. This framework controls the execution of the models and a myriad of other functions distributed to a variety of UNIX computers, allowing each application to take advantage of the computer best suited to meet its needs. 
The complete elimination of dependent manual steps is another requirement of ARAC's emergency response system. A series of automatic actions are performed based on the information stored about a particular event. The system generates an initial calculation in a "hands-off" response mode (i.e. no human intervention is required) and in a TAHOE (Totally Automated Hands Off Exercise) mode. The former is the foundation of ARAC's rapid initial response while the latter allows ARAC's remote client sites to practice exercise responses with ARAC assessment plots as frequently as they wish. These steps include activities such as activating the execution of the model, obtaining metdata, and producing final plots. Part of the model execution process determines what models to execute, on what computer system to execute the models, where to direct the output, and how to monitor the modeling process.

Data acquisition and communications involves obtaining data from outside sources as well as communicating with ARAC's remote sites. The ARAC System communicates with remote ARAC site systems through several network communication paths (dial-up, DOE Emergency Communications Network, InterNet and private leased lines). The standard communication with an ARAC remote site system involves transferring problem definition information, metdata, field measurements, model products, and mail. Periodic communication with the sites is required to maintain metdata acquisition from the meteorological towers, upgrade software and hardware, maintain up-to-date information on the individual site's environment, and track any problems with the system. Other remote communications include FAX communications between the sites/clients and the ARAC System, and connecting with an outside news wire service. The acquisition of metdata involves acquiring data from AFWA (Air Force Weather Agency), FNMOC (Fleet Numeric Meteorology and Oceanography Center), NOAA's DDP (Domestic Data Plus) and IDS (International Data Services), ARAC remote client site systems, meteorological towers, commercial weather data suppliers, and the Internet. This metdata includes both observational data and gridded data. New sources of metdata as well as new types of metdata will be acquired in the future.

A rich collection of data is required to satisfy the expanded scope and capabilities of ARAC. The source of that data consists of both external and internal data. External data includes worldwide weather information and cartographic suites. Internal data includes information that implements the knowledge base of an emergency response system as well as data required to operate the system models. Virtually all data stored within the ARAC system is accessed and archived through a collection of databases. Exploiting database technology to organize the 
diverse databases has simplified the application programs access to the varied information bases that are required to make a model prediction. Because much of the design of the nonmodel components of the system is based on the object oriented paradigm, an object-oriented database management system is used to manage this information.

Types of data (to be) stored in the database are: metdata, land surface geodata (geodata used directly in model calculations, e.g., elevation data), reference geodata [base map information, gazetteer data (geographic names), demographic data, etc., which is used to orient assessors and users geographically], nuclear power plant and facilities locations, chemical data, and radionuclide dose factor information. Metdata consists of both observational and gridded data. Global geographic data is needed in order to respond to events anywhere in the world on domains ranging from $\sim 2-20,000 \mathrm{~km}$. Land surface geo-databases include: ETOPO5, DMA Level I DTED, ARAC 500m \& 125m data, USGS DEM data, USGS 1:250,000 Land use/Land cover data, Soil Conservation Service STATSGO soil data and bi-weekly AVHRR Normalized Difference Vegetation Index (NDVI) data. Reference geo-databases include: NCAR Global \& US Maps, USGS DLG data, DMA DCW, Census TIGER data, DMA ADRG, hand digitized site facility maps and local regions, USGS Geographic Names Information System, DMA Gazetteer data, Census Bureau population data (or equivalent value-added databases) (9). Sources for the chemical database include: CAMEO, ALOHA, DIPPR, and ERPGs.

Visualization and Other Tools: A suite of tools provides access to the ARAC resources and supports an integrated decision-making capability. This involves simultaneous visualization of multiple data types (terrain, map features, observed metdata variables, analyzed metdata variables), availability of defaults which minimize re-typing of information (e.g., selection of updated site-input Questionnaire entries rather than having to re-enter these values), graphical methods for user input, continual monitoring of processes, etc. Tools manage both the standard system administrative functions as well as those unique to an emergency response. Some of these are provided as part of the operating system and some are specialized tools. The standard system administrative tasks involve daily, weekly, and monthly backups of both the user and system disks, disk optimization, performance monitoring, accounting information, maintaining both classified and non-classified systems, and providing security on the systems as well as individual elements of the system.

Network Interface: The ARAC Client System allows users (such as emergency managers and first responders) with commonly available desktop and laptop computers to utilize the central 
ARAC system over the Internet or any other communications link using Internet protocols. Providing cost-effective fast access to the central ARAC system greatly expands the availability of the ARAC capability. The ARAC Client system consists of (1) local client applications running on the remote user's computer, and (2) "site servers" that provide secure access to selected central ARAC system capabilities and run on a scalable number of dedicated workstations residing at the central facility. The remote client applications allow users to describe a real or potential emergency event, electronically send this information to the central ARAC system which performs model calculations, and quickly receive and visualize the resulting graphical products. The site servers support simultaneous access to ARAC capabilities by multiple users. The ARAC Client system is based on object-oriented client/server and distributed computing technologies using CORBA and Java, and consists of a large number of interacting components.

\section{JAERI/WSPEEDI}

The establishment of a full-scale off-site countermeasure program in Japan against accidental releases of radioactivity was initiated by the TMI-2 reactor accident in the United States in March 1979. In the next year, JAERI began a research project designed by the Japan Nuclear Safety Commission (JNSC) to develop technology to assist experts of national and local emergency headquarters in assessing the environmental impact of accidental releases. In 1986, this project yielded a computer-based dose prediction system SPEEDI (System for Prediction of Environmental Emergency Dose Information) ${ }^{9}$, the predecessor of WSPEEDI. SPEEDI is now available at the Nuclear Safety Technology Center (NUSTEC) as a national emergency response system for the real-time prediction of atmospheric dispersion of radionuclides in domestic local emergency situations.

The Chernobyl nuclear accident in the same year necessitated the expansion of the computational area of SPEEDI to evaluate long-range transport of airborne radioactivity due to a severe nuclear accident in a foreign country. In response to this requirement, a new technical capability, WSPEEDI, has been developed to predict the radiological impacts on the Japanese people of a nuclear accident abroad ${ }^{10}$. Presently, WSPEEDI includes the capability of SPEEDI, and can respond to the various-scale dose assessment against domestic and overseas nuclear incidents.

The response domains of WSPEEDI are typically "local" and "regional" scales (25 to $100 \mathrm{~km}$ ) for domestic incidents, and "synoptic" (a few thousand km) and "hemispheric" scales for foreign 
ones. Forecast periods for domestic and foreign incidents are 48 hours and 7 days, respectively. An accidental discharge at any place in the world can be treated, assuming a point source including a maximum of five nuclides with temporal variation of release amounts. Although WSPEEDI operated at JAERI does not provide 24-hour service, it can respond immediately during the working time of JAERI.

The above capability is realized by various supporting functions, combined with threedimensional atmospheric models, e.g., data acquisition network, databases, graphical user interface (GUI) and graphic software for output. The input data are divided into two types, i.e., time-dependent data like meteorological and release information and invariant data. Meteorological data and source information are input in real-time from a data acquisition network and a keyboard, respectively. Meteorological data consist of gridded numerical forecasts from Japan Meteorological Agency (JMA) over Japanese (RSM) and Global (GSM) areas, and observations around nuclear power plants in Japan. Invariant data are extracted from the databases. The databases consist of map data, site data, nuclear physical constant data and isotopic composition data. Map data of Japan and the World are from the Digital Map of Geographical Survey Institute and ETOPO30, respectively. Physical constant data include decay constants and dose conversion factors for 60 radioactive nuclides. Isotopic composition data are isotopic ratios of noble gases and iodines at shutdown. The data in databases are extracted automatically based on the input of source information for an accident site, discharged nuclides, etc. The system operation, e.g., input of source information, execution of predictions, plotting of results, etc., is carried out through a Motif based GUI which minimizes input parameters for quick response. Predicted results of 3-D wind fields, 3-D airborne concentration, 2-D deposition, 2-D external and internal doses are output as binary files and plotted in the form of 2-D wind vectors, contours, temporal variations at arbitrary points or 3-D features by AVS superimposed on a map. Then, these plots are stored in graphical databases which can be accessed by a Web browser.

The atmospheric models of WSPEEDI are PHYSIC, WIND21, PRWDA21, WSYNOP and GERAN. A suite of models for domestic incidents consists of three models ${ }^{11}$, a hydrostatic meteorological forecast model PHYSIC, a mass-consistent wind field model WIND21 and a Lagrangian dispersion model PRWDA21. These three models are formulated in the terrain following $z^{*}$ coordinate system. The horizontal coordinates are Cartesian coordinates. PHYSIC simulates regional three-dimensional meteorological fields, e.g., wind, potential temperature, 
and turbulence characteristics, by numerically solving a set of atmospheric dynamic equations with hydrostatic assumptions. WIND21 serves to eliminate some mass imbalance which will be caused by the conversion of regional data to "local" coordinates with a finer resolution topography. WIND21 has two successive procedures, i.e., interpolation of regional wind fields onto local grids and diagnostic analysis to adjust interpolated wind fields to mass-consistent ones. PRWDA21 models the atmospheric dispersion of radioactivity by following the trajectories of a large number of marker particles, discharged from a source and moving in the downwind direction. The concentration at each unit Eulerian cell is calculated by summing up the contribution of each particle to the cell.

PHYSIC has been examined for the purpose of introducing the forecasting function of local meteorological conditions around the site. Forecast wind speeds and directions at the stack are compared with those at several nuclear power plants. In the case when the error of 22.5 degree of wind direction is allowed, $70 \%$ of forecasts on wind direction agreed with measurements for $24 \mathrm{hrs}$. WIND21/PRWDA21, the combination of models, has been applied to datasets from a series of field tracer experiments on the east coast of Japan with flat terrain, around isolated conical mountain regions and in mountain-valley areas. The accuracy obtained from the verification studies is a factor of 10 for $50 \%$ of samplers for complex terrain and 2 for flat terrain near the seacoast.

For domestic responses, synoptic meteorological forecasts over Japan, RSM, are released on-line by JMA twice daily. It provides WSPEEDI with a synoptic forecast of wind, temperature, precipitation, etc., initialized at 09 and 21 JST (Japanese Standard Time). The forecast period is 48 hours, with a 6-hour output interval. The data cover Japan by a mesh with the horizontal resolution of $20 \mathrm{~km}$ near the surface and $40 \mathrm{~km}$ in the upper air. When an accident occurs, PHYSIC starts the regional meteorological forecasts with the grid size of $50 \times 50 \times 30$, with $2 \mathrm{~km}$ resolution horizontally. RSM is used for the initial condition at 09 or 21 JST just before the accident and the boundary conditions. Furthermore, PHYSIC includes a data assimilation technique to incorporate the observed meteorological data into the model equations as external forces. The output is a regional forecast of wind fields, diffusion coefficients and precipitation. The prediction period is for a maximum of 48 hours, with output every hour. Following the forecast by PHYSIC, hourly wind fields and diffusion coefficients from PHYSIC are interpolated on the three-dimensional grid of WIND21. WIND21 starts diagnostic analysis of the "local" wind fields and diffusion coefficients with a finer mesh (50 ×50 × 20 with $0.5 \mathrm{~km}$ resolution) than that 
of PHYSIC, considering the emergency planning zone of $10 \mathrm{~km}$ recommended by the JNSC. Then, the dispersion of radionuclides and the radiological doses are calculated by PRWDA21, by using wind fields and diffusion coefficients from WIND21. Here, the grid size of PRWDA21 is $100 \times 100 \times 20$, with $0.25 \mathrm{~km}$ resolution. PRWDA21 can also be connected with output from PHYSIC directly for regional predictions with $1 \mathrm{~km}$ resolution.

The long-range dispersion models for overseas incidents still consist of two models, a massconsistent wind field model WSYNOP 12 and a Lagrangian dispersion model GERAN ${ }^{13}$. Although the modeling concept of these models is similar to WIND21 and PRWDA21, several points are improved for long-range transport, e.g., inclusion of vertical flows induced by low/high pressure systems, distribution of precipitation for wet deposition and horizontally transformed map coordinates. Regarding meteorological forecasts over synoptic and hemispheric areas, WSPEEDI completely depends on GSM from JMA.

Because the area of concern will depend on the location and scale of the accident, WSPEEDI has a preprocessing function to extract the geographical data for the relevant region from the master database which contains worldwide geographical data. The grid size and number are determined depending on the impacted region. The global meteorological forecast data, GSM, are released with RSM by JMA twice daily. It provides WSPEEDI with a global forecast of wind, temperature, precipitation, etc., which are initialized at 09 and 21 JST (Japanese Standard Time). The forecast period is 192 hours, with a 6-hour output interval. The data cover the globe by a mesh with the resolution of $2.5 \times 2.5$ degrees horizontally. When an accident occurs, horizontal wind fields from GSM are interpolated on the three-dimensional grid points of WSYNOP. WSYNOP starts diagnostic analysis of the 3-D wind fields over the impacted region, e.g., Asia, Europe, etc., with high-resolution topography. Then, GERAN calculates the dispersion of radionuclides and the radiological doses by using wind fields from WSYNOP. Here, since the information from WSYNOP lacks the spatial and temporal variation of the boundary layer and turbulence strength, a homogeneous boundary layer with neutral atmospheric condition is simply assumed for vertical diffusion.

The computer programs of these models are being revised for a vector parallel processor, VPP-500 of Fujitsu Ltd. for the quick response. It is expected to shorten the computation time for, particularly, hemispheric simulations. 
In parallel to the development of the system, JAERI has conducted validation studies, exercises and responses to real events during the last decade, e.g., model evaluation studies ATMES and ETEX organized by the EC, WMO and IAEA, the international nuclear emergency exercise INEX by OECD/NEA, a fire accident of a bituminization plant for radioactive waste at the former Power Reactor and Nuclear Fuel Development Corporation (PNC) at Tokai, Japan, an accidental discharge of Cs137 in Algeciras, Spain, the JCO criticality accident at Tokai, Japan and the continuous discharge of huge amounts of volcanic gases $\left(\mathrm{SO}_{2}\right)$ from the Miyake Island, Japan. Through such experiences, three state-of-the-art functions needed for a future WSPEEDI were identified and are under construction. These are: (1) a prototype international data communications network to exchange information on nuclear accident emergency situations; (2) a synoptic hydrodynamic model to forecast detailed meteorological conditions; and (3) a source term estimation on the release point, time and amount by coupling monitoring data with atmospheric simulations. 


\section{PROTOTYPE COMMUNICATIONS PROTOCOL}

The technology of the prototype communications link can be broken into two components hardware and software. Furthermore, the hardware of the system can be considered as two major components - the computer platform on which the system runs, and the network over which information is exchanged. Initially, a Silicon Graphics Indy workstation serves as the platform at both ends of the connection. Figure 1 shows an SGI Indy workstation and whiteboard in the display. The Indy has a $133 \mathrm{MHz}$ processor in it, and is capable of processing the current data throughput. A 19" color monitor is essential for displaying numerous concurrent windows containing often complex color graphics. After some early tests, we found it necessary to upgrade the memory from $96 \mathrm{Mb}$ to $160 \mathrm{Mb}$ to accommodate the numerous large visual buffers generated by the Web browser and video- teleconference applications during a typical session. Also, the Indy was soon running out of disk space (due to lack of swap space), so a second local disk was also added to the workstation. With these improvements, the Indy functioned well during real-time sessions, even with the Web browser running and several active whiteboards. An ISDN connection was also added in preparation for possible future applications. This will be discussed in the next section. Other required hardware includes a microphone and a video camera for capturing these portions of the session for transmission to the other conference participants.

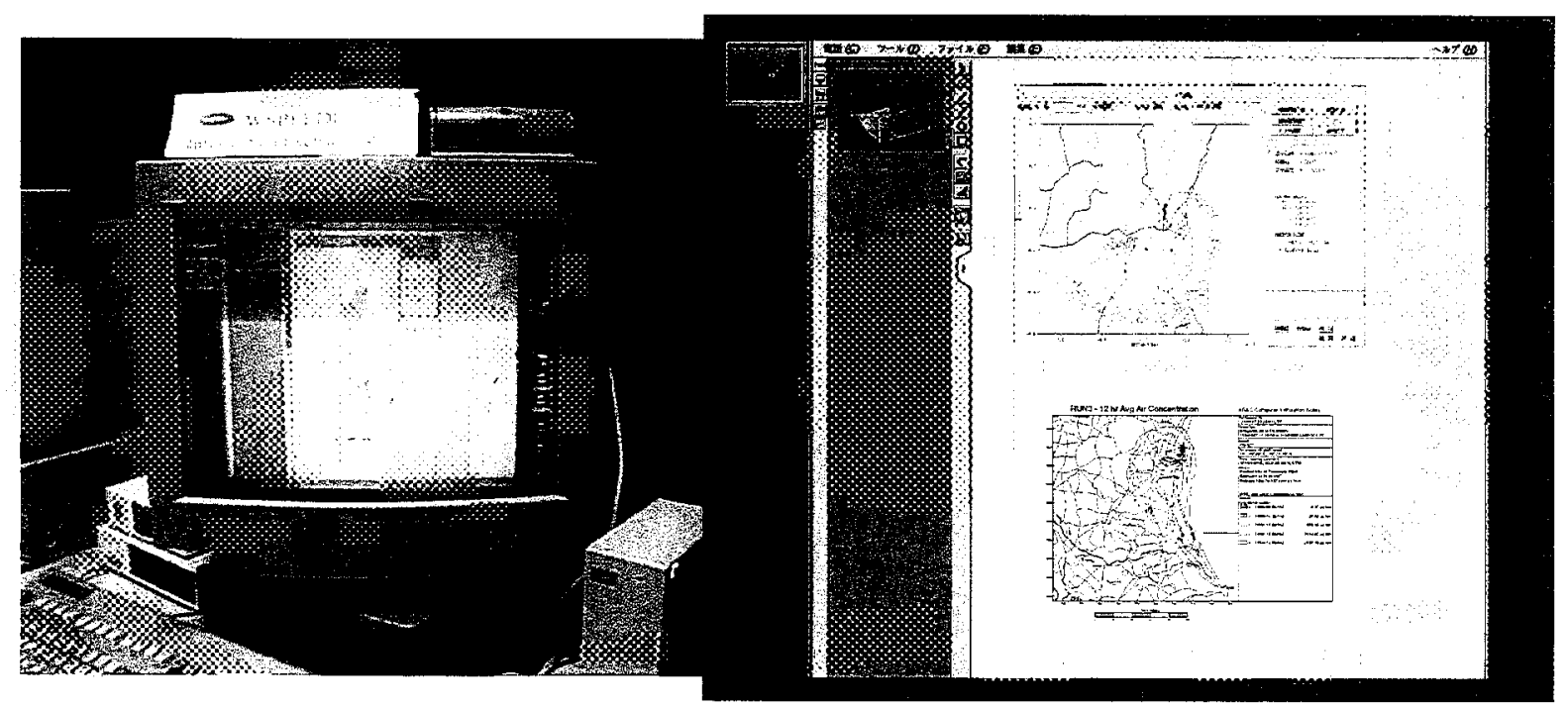

Figure 1. Silicon Graphics Indy Workstation with video camera and microphone. White board for teleconference is shown in the display. 
At the outset of the project, several network options were considered for the videoteleconference portion of the session. The first option was the standard Ethernet connection to the Internet, also used by the Web browser. There was concern about the unreliability of the bandwidth, especially during windows of peak Internet usage. This problem in fact did show up early in the testing, and it became necessary to disable the video portion of the videoteleconference tool as standard practice in order to allow transmission of the audio and whiteboard data. To date, this is the only network option actually tested with real data, mainly because of budgetary constraints. Other options considered were: 1) a dedicated point-to-point leased line, which is currently unrealistic because of cost; 2) ESNet, a possibly more reliable (as far as guaranteed bandwidth) option, but impractical for JAERI because of inaccessibility; 3) ISDN, which has begun to be tested locally, though a US- Japan connection has not yet been tried. This has the potential of providing guaranteed $128 \mathrm{~Kb}$ throughput, and could possibly accommodate the video signal on one channel $(64 \mathrm{~Kb})$, while the audio and whiteboard are transmitted on the other.

The software for the project can also be considered as two components, the Web site/browser portion and the video-teleconferencing tool. For the Web site itself, where the actual computational data and graphics is posted and exchanged, a number of password-protected pages were written in CGI and HTML. Each Web site permits privileged users to log on and review the computational results for a given facility. Accessible information includes model parameters and assumptions used in the calculations, graphical displays of wind data, and plots of predicted concentrations, dose and deposition. These plots and data can be easily extracted and annotated for use on the white boards in scheduled teleconferences. Both Netscape and Explorer have been used as Web browsers for the project, and both have proven satisfactory for accessing, viewing and saving information from the remote Web sites. A Web browser is also typically used during a conference, as reference is constantly being made to information on the Web which may not be included in the white boards. Figure 2 shows the cover-pages of Web sites to provide the partner with prediction results, etc. 


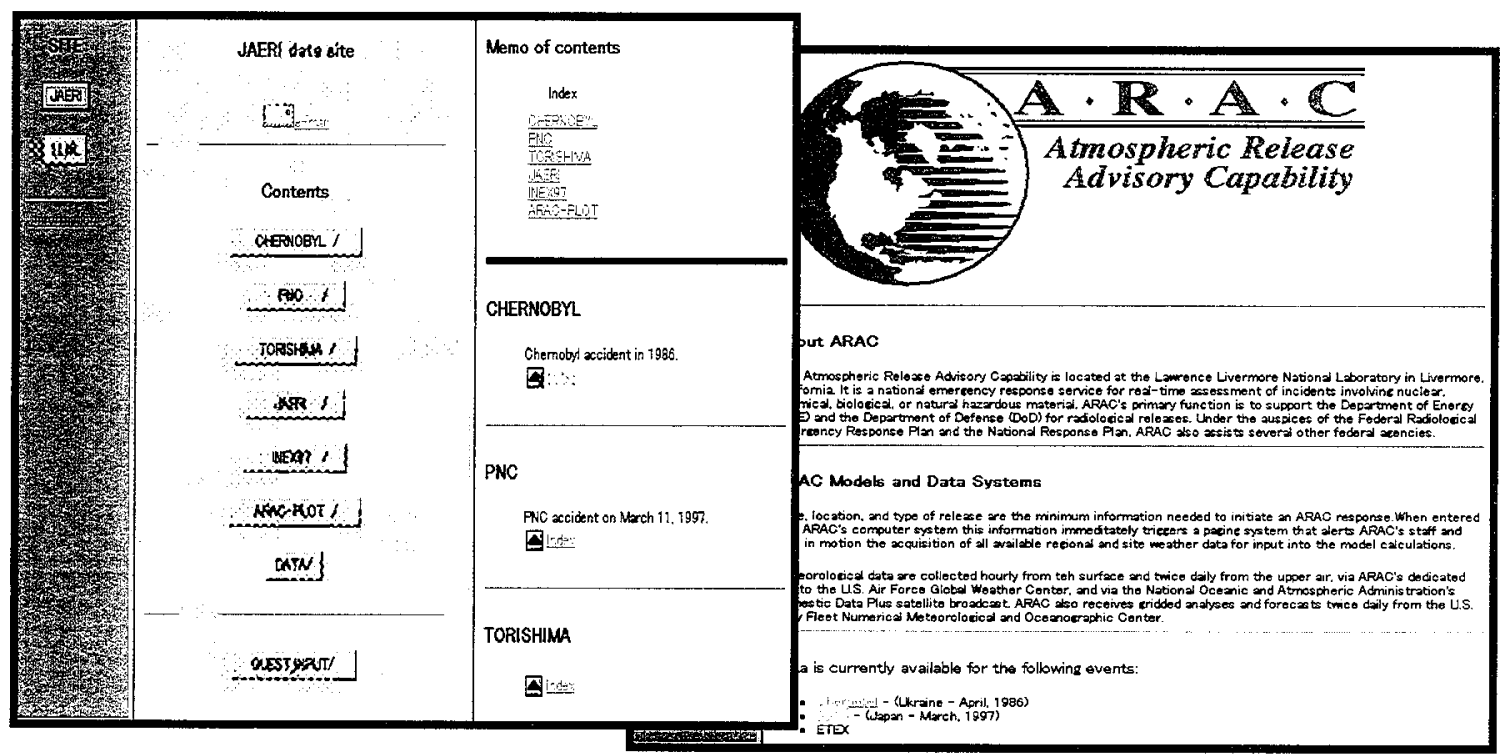

Figure 2. The coverpages of Web sites to provide the partner with prediction results, etc.

(Left: WSPEEDI, Right: ARAC)

The final component of the system to be considered is the video-teleconference tool. As in the case of the network itself, several options were considered at the outset of the project. The SUN product, ShowMe, was briefly considered, but was soon abandoned when the decision was made to go with Silicon Graphics workstations. When the SGI decision was made, the clear choice was InPerson, a video-teleconferencing tool which comes bundled with the SGI workstation. Like most video-teleconferencing tools, InPerson consists of three components-a video handler, an audio handler, and a whiteboard application. Each component is highly configurable and is easy to use. Both the video and audio components allow variable sampling rates for each signal to reduce the bandwidth required by each. As mentioned above, it became necessary to freeze the video signal completely because of its tendency to degrade the other signals when in use. The default ISDN-like bandwidth of $128 \mathrm{~Kb}$ setting was used, with total bandwidth set at $100 \mathrm{~Kb}$. This allowed a clear audio signal to be transmitted, though a delay of 5-10 seconds was experienced. This delay was not due to bandwidth per se, however, but to 
unavoidable Internet congestion problems at the various bridges and gateways between the US and Japan. A trace of the route revealed individual node delays of up to 5 seconds during peak Internet usage. Similar whiteboard delays were also experienced, but the overall performance of the system was acceptable. The gain of interactive data exchange, including real-time display and annotation of figures as well as concurrent verbal discussion were invaluable. The results accomplished over a two-week period in a cooperative response to an actual event (fire at PNC) would have been impossible to achieve using conventional exchanges via phone and e-mail. The combination of the Web pages and the teleconferences yielded a collaborative effort which could only have been otherwise achieved by actual face-to-face meetings. In fact, this prototype system even provides an advantage over the face-to-face exchange, as each participant is acting from their own institutional environments, where all local data and even colleagues are readily accessible, whereas the traveler must reduce their tools and information to a suitcase.

After evaluating and utilizing the SGI/InPerson combination for two years, two factors compelled a re-evaluation of the video-teleconference tool. These factors were: 1) Internet congestion during a real accident causing serious degradation of the connection and 2) the plan to expand the project to include collaboration with other countries where PC-based solutions are more readily accessible than workstations like SUN and SGI. The current package being tested is the PC-based tool ClearPhone, a product of Internet Communication Technologies Corporation. Though ClearPhone is primarily a Mac-based product, there is also a PC version available. Figure 3 shows teleconference between JAERI and LLNL using Mac-based ClearPhone. The advantage of this product over InPerson and many other products is not only that it is available on PC's, but the protocol used to exchange packets between conference hosts is designed to work well during times of peak Internet usage. We are currently evaluating the product. Several tests between LLNL and JAERI have indicated easier establishment of connection, more robust connectivity after session initiation, and better video and audio performance when headsets are used by both parties. The tool allows both textual and graphical data exchange, but may not permit interactive whiteboard annotation as easily as InPerson. The product is still under evaluation. 


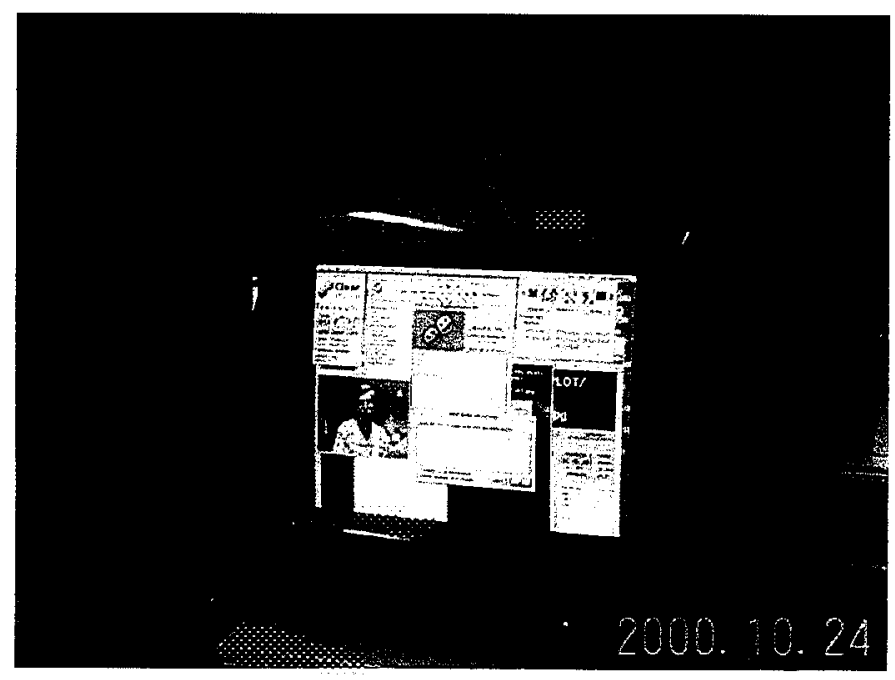

Figure 3. Teleconference between JAERI and LLNL using Mac-based ClearPhone.

Subsequent to the exchange of measurement data and products following the Algeciras event, JAERI and ARAC determined that the prototype communications protocol effort was successful enough to extend the effort to a third party. The EU funded RODOS program under development management at Forschungszentrum Karlsruhe Gmbh (FZK) was invited to join in the venture. In a preliminary meeting at FZK in the summer of 1998 an informal agreement was decided and the necessary formal arrangements determined. LLNL/ARAC and FZKJRODOS had in place a multi-year collaboration agreement. JAERI started the process to create a similar agreement which was concluded in the winter of 2000-2001. The triangular prototype communications protocol shown in Fig. 4 was presented to the full RODOS Consortium Workshop in September 1999 and received concurrence to proceed. At this workshop the three parties began the drafting of a formal data-exchange data structure document. This was proved necessary by the tedious time consuming and error-prone manual format conversions required in the three data exchanges and event comparisons which are described in the next section. Format structures were proposed and negotiated for placement of all the key data on protected web pages such that any of the parties should be able to readily read and ingest the information directly into their respective systems. It is anticipated that these data structures will be implemented this year (2001). 


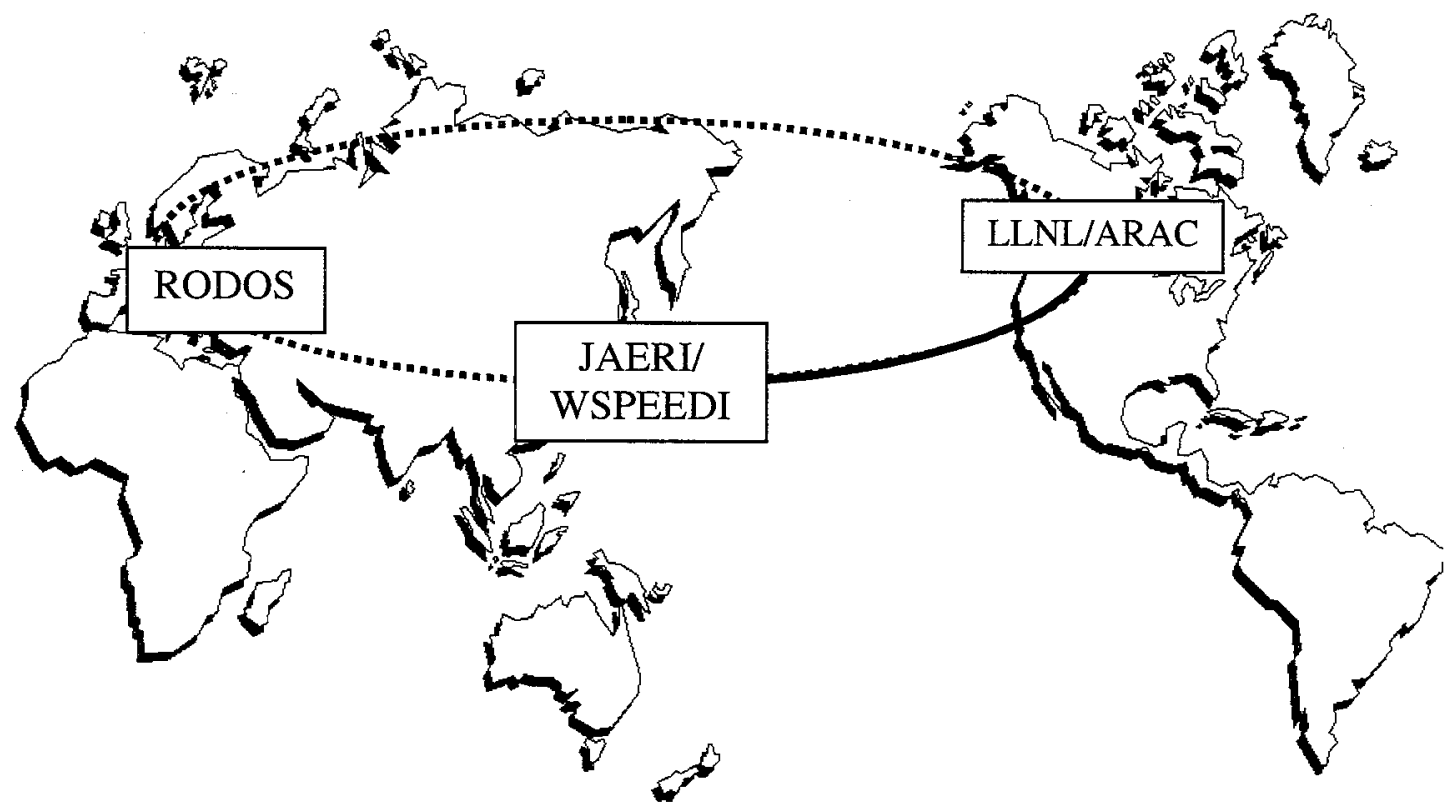

Figure 4. The triangular prototype communications protocol. (Dotted line is under planning) 


\section{APPLICATIONS}

\section{The former PNC fire accident at Tokai, Japan in 1997}

On 11 March, 1997, a bituminization plant for radioactive waste at the former Power Reactor and Nuclear Fuel Development Corporation (PNC) in Japan caught fire at 10:06 and exploded at 20:04 JST with the discharge of various radionuclides into the environment. Although in the early stage the former PNC stated there was no discharge of radionuclides into the atmosphere, Cs134 and Cs137 were detected after several days from dust samplers around the PNC and at Tsukuba, $60 \mathrm{~km}$ south of the PNC. Shortly thereafter ARAC and JAERI started cooperative work to estimate atmospheric dispersion of Cs137 in the area of about $100 \times 100 \mathrm{~km}$ and were able to view each system's model assessment plots. However, since both simulated using their own meteorological data, e.g., US Air Force Data for ARAC and GPV for SPEEDI, the first results were different as shown in Fig. 5.
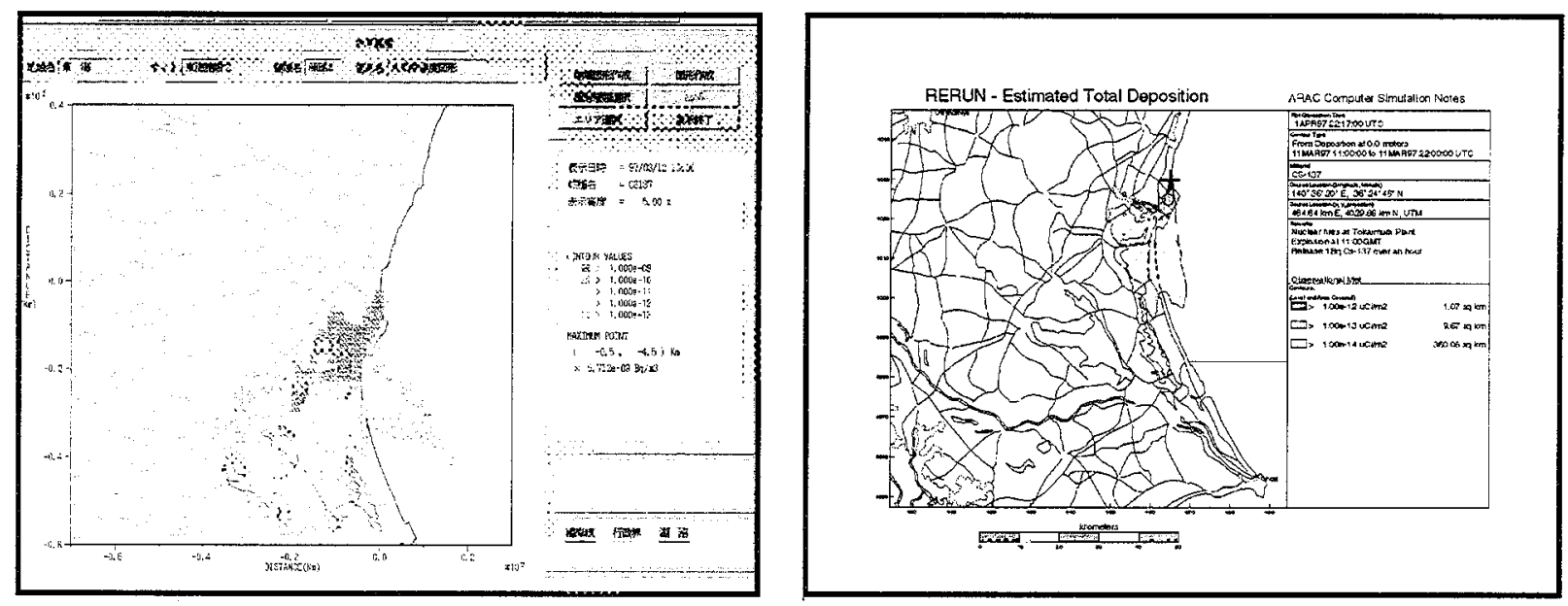

Figure 5. First predictions of accumulated air concentrations for $24 \mathrm{hrs}$ from WSPEEDI (left) and ARAC (right).

Subsequently, we discussed differences due to models and input data, accurately located measurements sites and data values and then re-compared and discussed results again when comparable data were used in both systems. In particular, because radionuclides were 
discharged into the strong stable atmosphere in the night, local meteorological data were very important for accurate simulations. Local data were provided from JAERI to LLNL and, finally, our results agreed well as shown in Fig. 6.
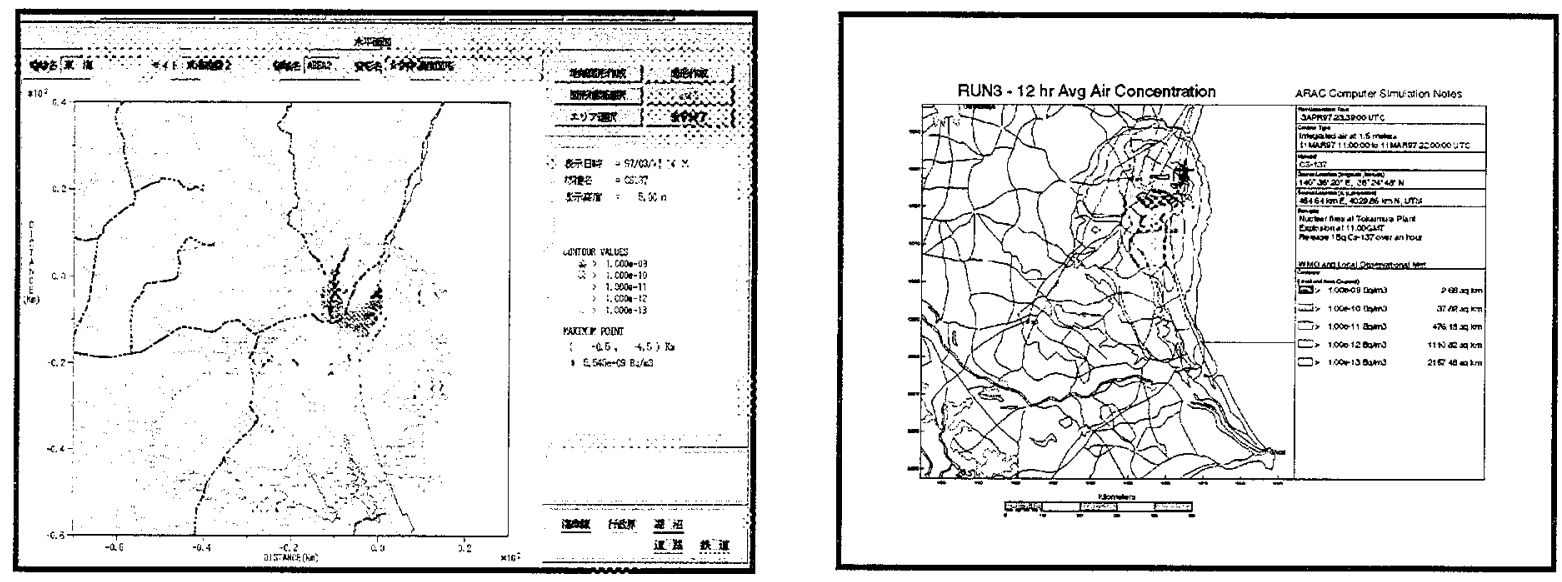

Figure 6. Final outputs of accumulated air concentration of Cs137 from WSPEEDI (left) and ARAC (right).

Both results revealed the complex processes of atmospheric transport during the night time and predicted accumulated air concentrations covered all the sampling points where Cs137 were detected.

The common activities included the estimation of the release amount of Cs137 by comparing predicted air concentrations with observed ones ${ }^{14}$ as well as the estimation of the contamination area shown by accumulated air concentrations. The release amount was estimated $1-2 \times 10^{9} \mathrm{~Bq}$ and reported to the national surveillance committee for the PNC accident.

\section{Accidental discharge of Cs137 at Algeciras, Spain in 1998}

An accidental discharge of Cs137 occurred at Algeciras, Spain on 30 May, 1998, was the second cooperative response to real events. In the extreme southern tip of Spain, near the town of Algeciras, an accidental release of cesium provided an opportunity for ARAC to use actual, if sparse, measurements of a dispersion event to back-calculate the magnitude and extent of the original release. 
On June 9, 1998, the Swiss government announced that radiation levels up to a thousand times background had been detected by their national monitoring network. The source was unknown. France and Italy also reported measurements. The next day, a steel mill near Algeciras notified the Spanish Nuclear Security Agency that radiation had been detected in one of its oven filtration systems. The agency, however, had not observed elevated radiation levels in its national network. On June 12, the source of the release was identified as a medical radiotherapy device containing Cs137 that apparently melted in the steel mill's scrap metal furnace. The amount and time of the release were unknown, but the incident was thought to have taken place during the last week of May 1998. On June 12, the International Atomic Energy Agency also announced the incident and speculated on its possible connection to elevated levels of Cs137 detected at the end of May and early June in southern Europe.

As ARAC staff became aware of the incident, they extracted meteorological data archived for the area and began acquiring preliminary ambient cesium air concentrations measurements from European colleagues. The various countries offering data had collected them with different samplers, using different averaging times (ranging from 1 to 14 days) and different radiological sensitivity thresholds. Having only data of sparse and varying quality, the staff set themselves the challenge of modeling the incident without knowing the exact location of the release. With each successive simulation, they incorporated new data as they were received and made model adjustments based on what they learned from previous simulations.

The first simulations led to an estimate of a $100 \mathrm{Cp}(3,700 \mathrm{GBq})$ release of $\mathrm{Cs} 137$ over a 12 hour period on May 29, 1998. At that point, ARAC received more data from eastern and central Europe on concentrations of cesium in the air at greater distances downwind. ARAC enlarged the model domain to include these data. It also decreased the release duration from 12 hours to 30 minutes, based on results from the first model run. By the third simulation, the exact location of the steel plant releasing the cesium became known and was incorporated into the model, as were the parameters of the stack responsible for the release. Once this location was pinpointed, ARAC meteorologists blended observed meteorological data from the area with gridded weather data and produced their final simulation. It led to an estimate of a $50 \mathrm{Cp}(1,850 \mathrm{GBq})$ release, which compared favorably with the $8-$ to $80 \mathrm{Cp}(296-$ to $2,960 \mathrm{GBq})$ estimate provided by the Spanish government.

The Algeciras release was too small to produce any measurable health effects. However, the fact that ARAC tracked even this small release for great distances demonstrates the potential of 
accidental releases to affect substantial geographic area and populations. ARAC used the Algeciras release to refine and validate techniques and to further evaluate its ability to model on large spatial scales. The post-accident analysis validated ARAC's capability to effectively provide consequence assessments for a complex event of continental scale. 


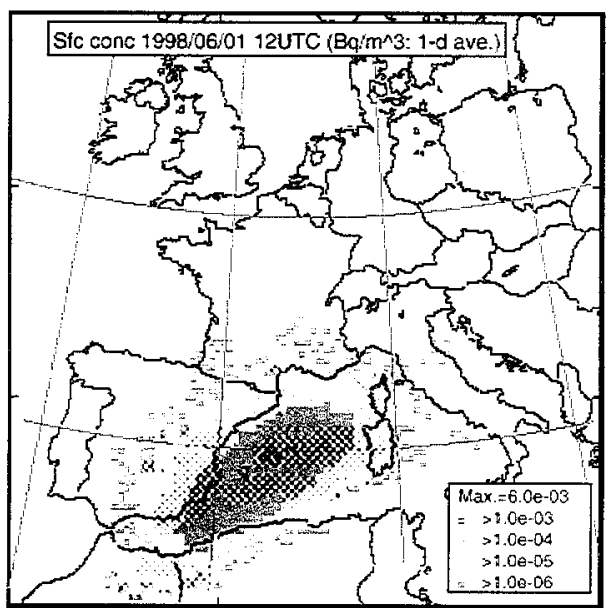

ARAC

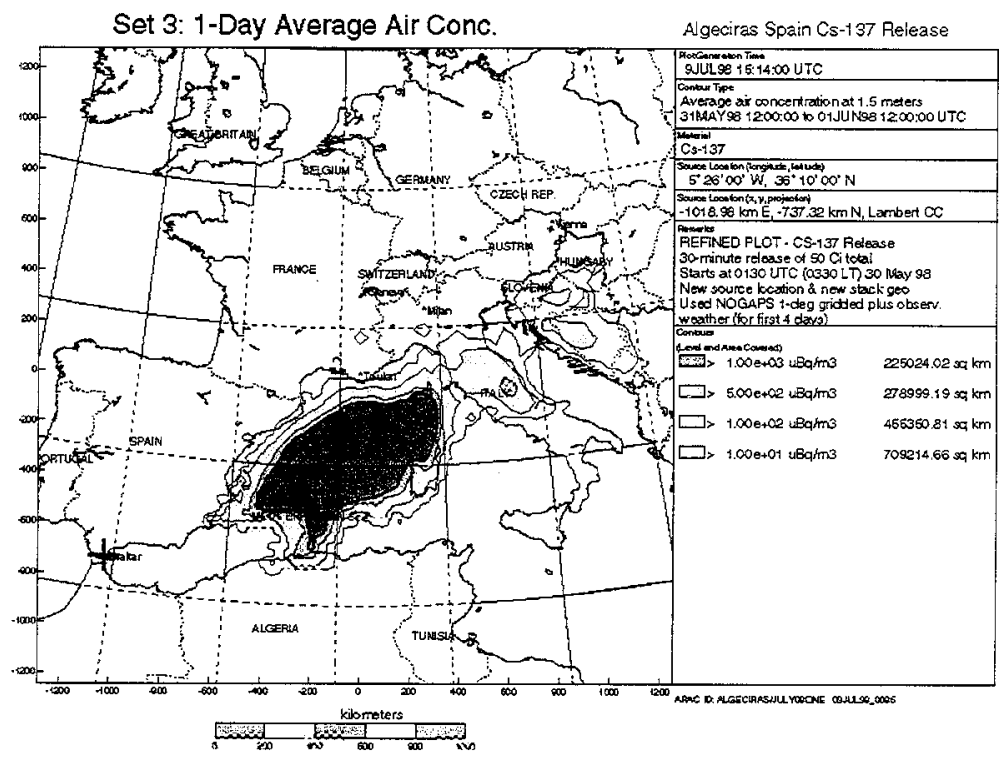

a.

Figure 7a, b. Comparison of results for the Algeciras accident, Spain. (24-hr Averaged air concentration of Cs-137 near the ground level.) 
WSPEEDI

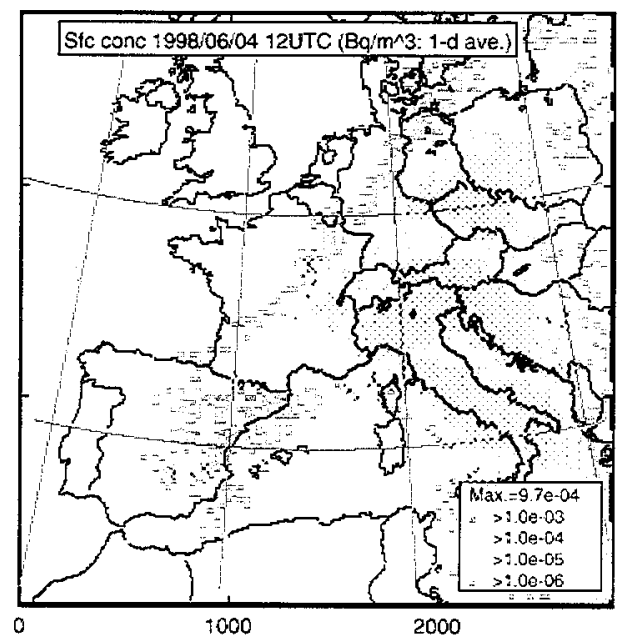

Set 3: 1-Day Average Air Conc.

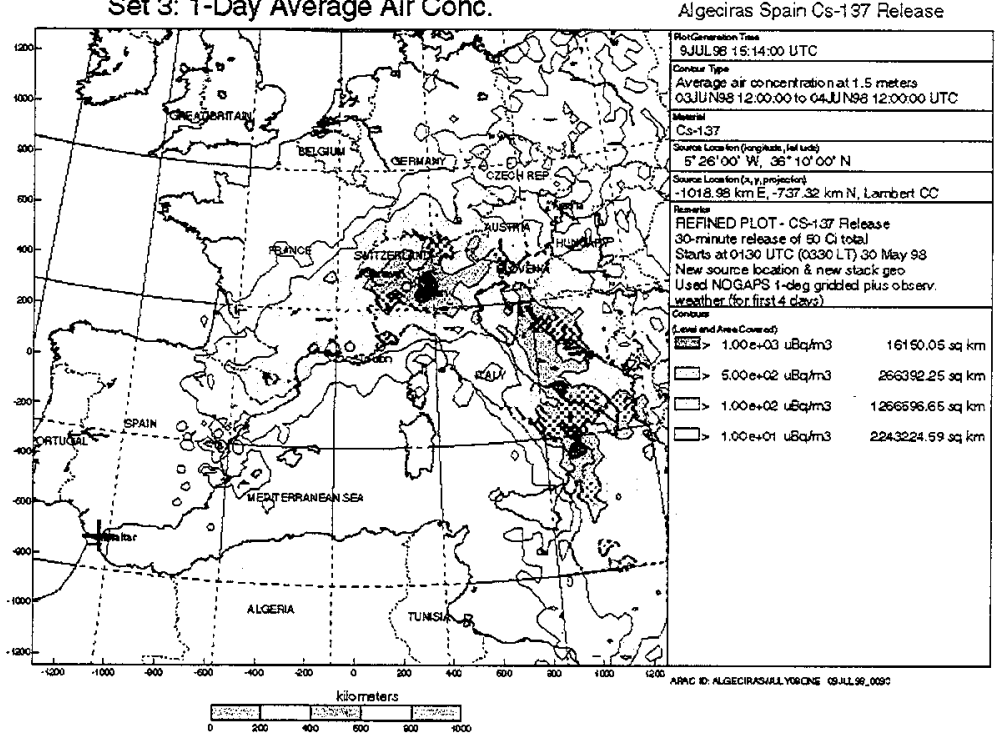

b. 
JAERI received accident information and monitoring data from LLNL and immediately predicted the atmospheric dispersion of Cs137 over Europe. This activity also included the estimation of accumulated air concentration and the release amount of Cs137 ${ }^{15}$. As JAERI received reliable data from LLNL, a simulation was carried out one time using meteorological forecast GSM. The comparison of air concentration of Cs137 at the ground level between ARAC and WSPEEDI is shown in Fig. $7 a$ and b. Both results agreed well. The estimated release amount of Cs137 was 60 Ci over a 12-hour period on May 29, 1998.

\section{The JCO criticality accident at Tokai, Japan in 1999}

On 30 September 1999, a criticality accident occurred in the conversion building at the nuclear fuel processing facility, JCO Company in Tokai, Japan. Just after the accident, the $\gamma$ dose rates increased at the several monitoring posts at the vicinity of JCO and it then returned to the background level. However, at about 16:00, when the wind direction began to change, elevated $\gamma$ dose rates were measured at many monitoring points at the east side of $\mathrm{JCO}$ within $10 \mathrm{~km}$. It is considered that the first increase was caused by direct $\gamma$-ray due to the criticality, and the second was from the radioactive plume of noble gases and iodine. In response to this accident, JAERI and LLNL engaged a strong cooperative effort to estimate the transport of the plume, the release amount and the radiological dose to the public. The starting day of cooperative work was still in a confused state and even JAERI could acquire only limited information, e.g., only release point, criticality period, some meteorological and radiological data. However, both tried preliminary atmospheric transport and the results agreed well. After several days, detailed meteorological and radiological data were arranged at JAERI and sent to LLNL. After accumulating common data, both repeated the calculation of dose evaluations. Calculation conditions were as follows:

(1) Objective region: $25 \times 25 \mathrm{~km}$ square around the JCO site. For atmospheric transport simulations, the region was divided into $100 \times 100$ grid cells with a horizontal resolution of $250 \mathrm{~m}$.

(2) Meteorological data: Meteorological forecasts over Japan initialized at 9:00 JST, 30 Sept. from Japan Meteorological Agency (JMA) and observed meteorological data around JCO.

(3) Release Condition: Continuous unit $(1 \mathrm{~Bq} / \mathrm{h})$ release of noble gases and lodine from 10:35 JST 30 Sept. to 6:00 JST 1 Oct. 
Concerning the daughter nuclides, Rb89 and Cs138 generated from Kr89 and Xe138 were considered. This was because the major nuclide detected from aerosols in the atmosphere was Cs138. The parent nuclides, Kr89 and Xe138, have high isotopic ratio, and $\gamma$-energies from both nuclides are high.

Analyzing the prediction of atmospheric dispersions, meteorological conditions during the accident were divided into four characteristic regimes. The first was a sea breeze from E or ESE with wind speed of $2-3 \mathrm{~m} / \mathrm{s}$ during the period from 10:00 to 17:00, 30 Sept. The second was a transient period from sea to land breeze around 17:00 to 18:00; the wind became almost calm and wind direction changed widely. The third period from 19:00, 30 Sept. to 3:00, 1 Oct. was a land breeze from the NE. The last was after 4:00, 1 Oct. when winds were strong and from the NE. During the first period of sea breeze, the radioactive plume was transported to the direction of WNW and this situation continued till 17:00. In the transient period, the week southerly wind transported the plume slightly to the north. After 20:00, the plume was transported to the seacoast area by the land breeze and covered the southeast area. This situation continued till 3:00, 1 Oct., and finally the strong northeast winds transported the plume to the southwest around daybreak.

The predicted air dose rates from WSPEEDI and ARAC are shown in Fig. 8. Both results well represent the movements of the plume mentioned above. The prediction results on air dose rates are compared with observed ones by Ibaraki Preficture and JAERI. Because the predictions were done under the assumption of unit release, the absolute values cannot be compared. However, the reliability of the predictions can be evaluated by comparison of the appearance time and the magnitude of peaks evident in the temporal variations of air dose rates at monitoring points. The comparison indicates that the plume passed between the posts at the north-west side during the daytime. During the period from sunset to midnight, several peaks due to the plume appeared at the posts in the area of east to south of the site. The predictions agreed well with the observations.

Since the HEPA filter was working at the ventilation duct and consequently discharged radionuclides were mainly noble gases, the air dose rates observed at the far area were due to noble gases. Thus, the ratio of observed values to predicted ones means the release rate of noble gases. The release rates are calculated by using the ratios of pair of the peaks from prediction and observation. The release rate estimated by JAERI is $8 \times 10^{12} \mathrm{~Bq} / \mathrm{h}$. The external doses due to noble gases can be calculated by the multiplication of the estimated release rate, 
$8 \times 10^{12} \mathrm{~Bq} / \mathrm{h}$, to predicted external doses under the assumption of $1 \mathrm{~Bq} / \mathrm{h}$ release. Figure 9 shows the horizontal distribution of external doses at a height of $1.5 \mathrm{~m}$ above the ground accumulated until 8:00, 1 Oct. The figure indicates that external doses were higher for the regions of the northwest and southeast which were downwind for long periods of time. The outside contour of the lowest value, $10^{-3} \mathrm{mSv}$, is comparable to the external dose from the background radiation for one day. According to the external doses estimated from observed air dose rates, only one observation at Funaishikawa exceeded $2 \mu \mathrm{Gy}\left(1.6 \times 10^{-3} \mathrm{mSv}\right)$ and this fact agreed well with Fig. 9. 


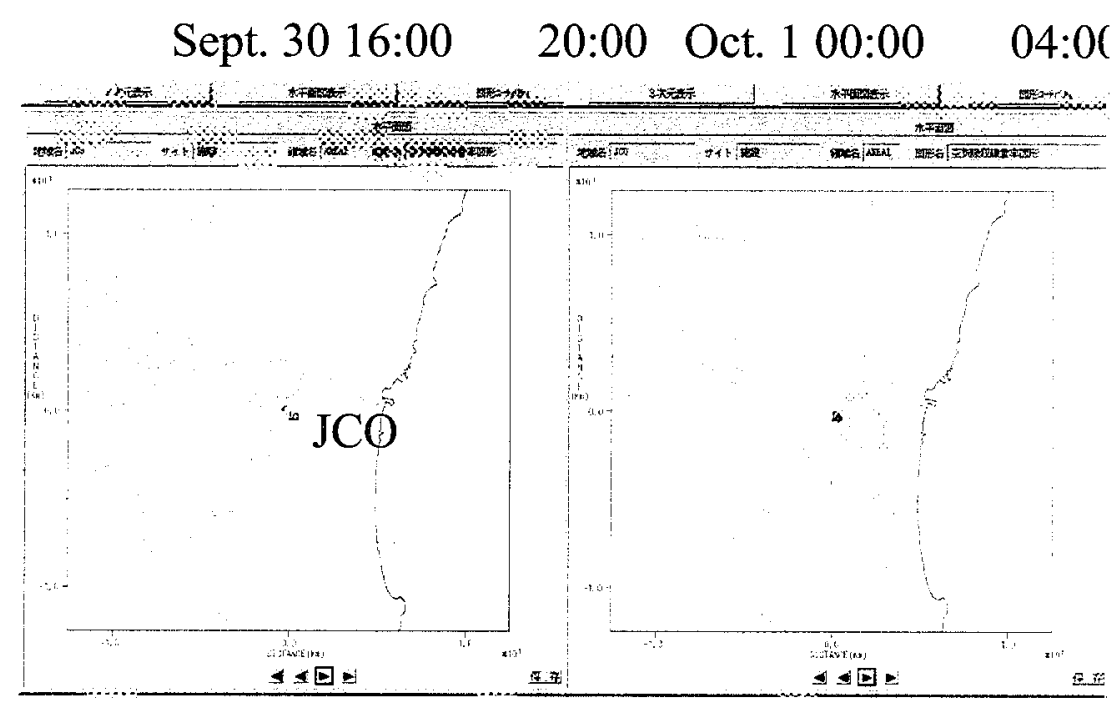

Sept. 30 16:00 20:00 (ARAC)
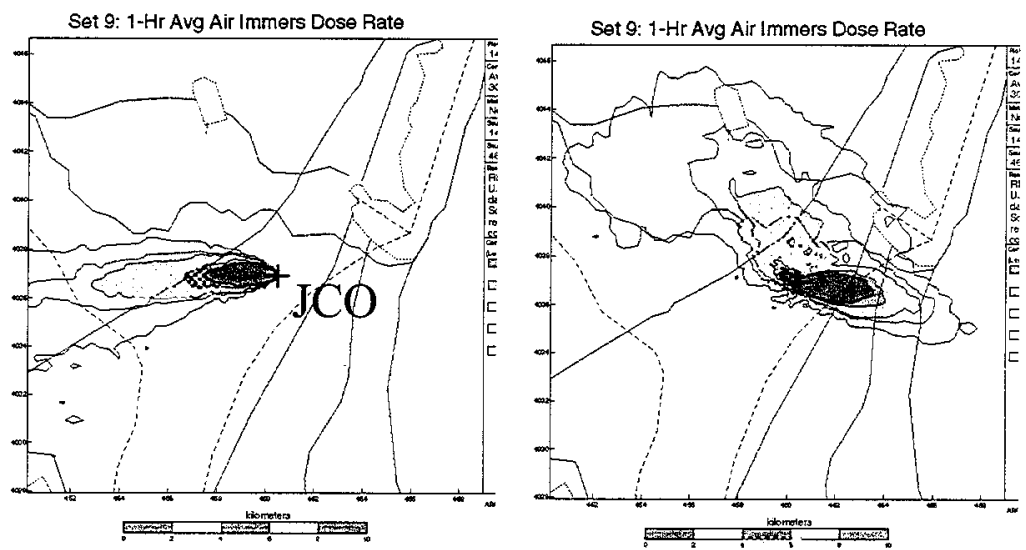

a.

Figure $8 \mathrm{a}$ and $\mathrm{b}$. Comparison of WDSPEEDI and ARAC hourly average air dose rates for the JCO criticality accident. 


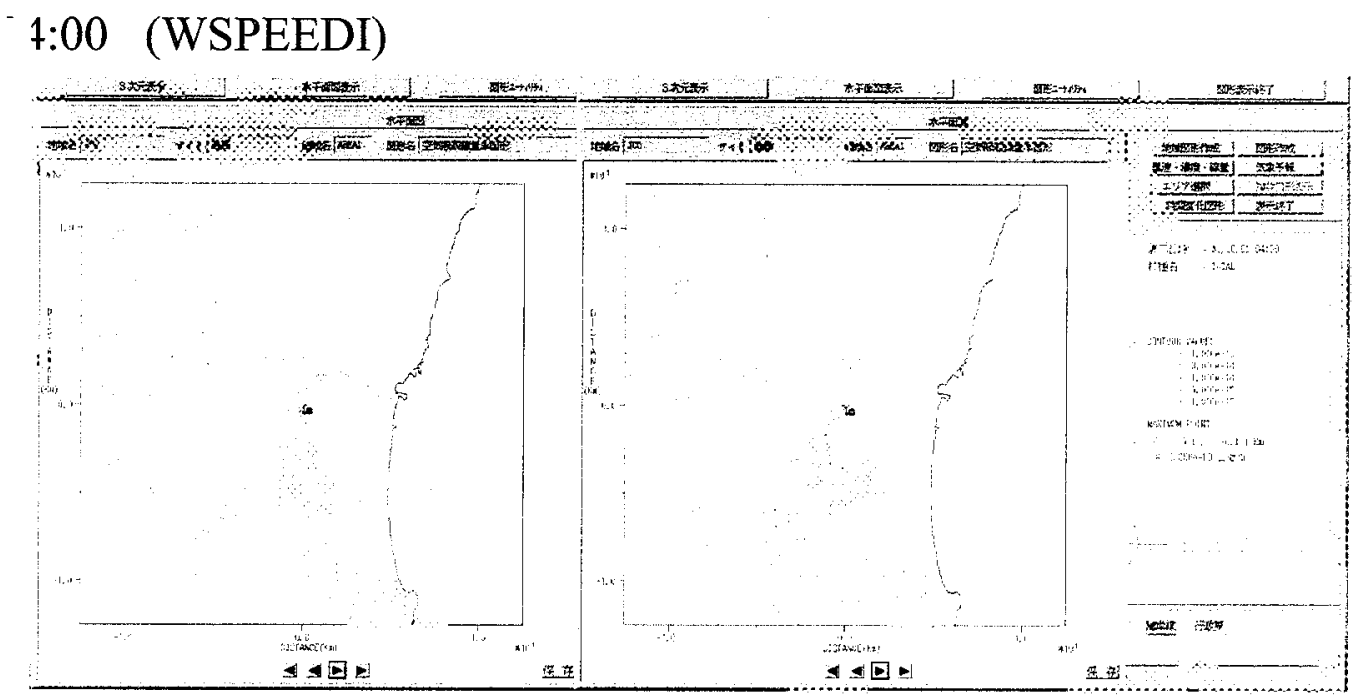

\section{J0 (ARAC)}
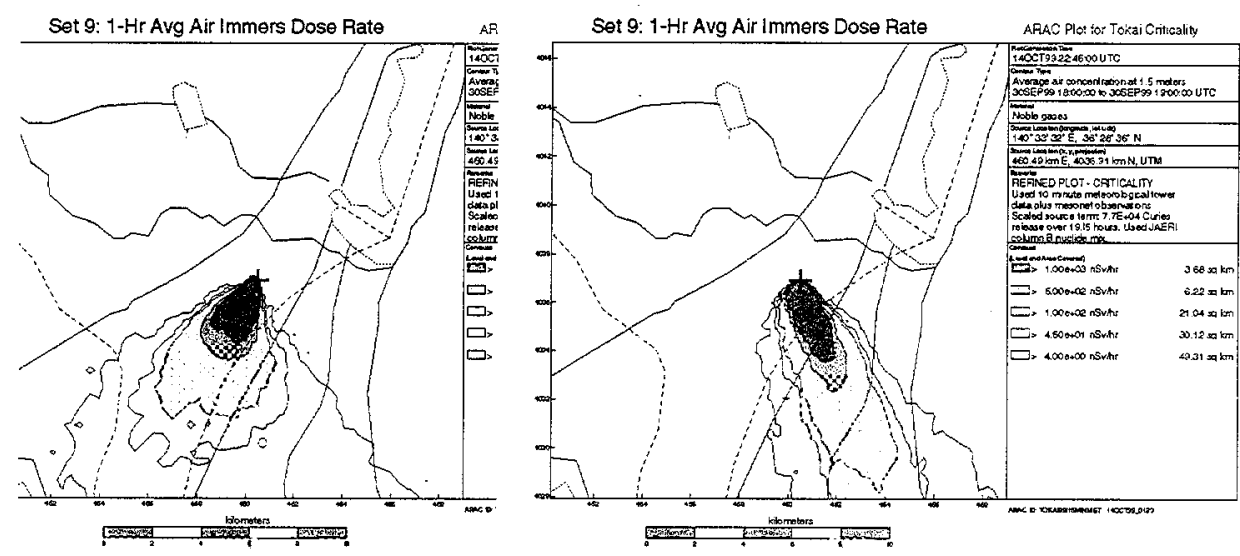

b. 


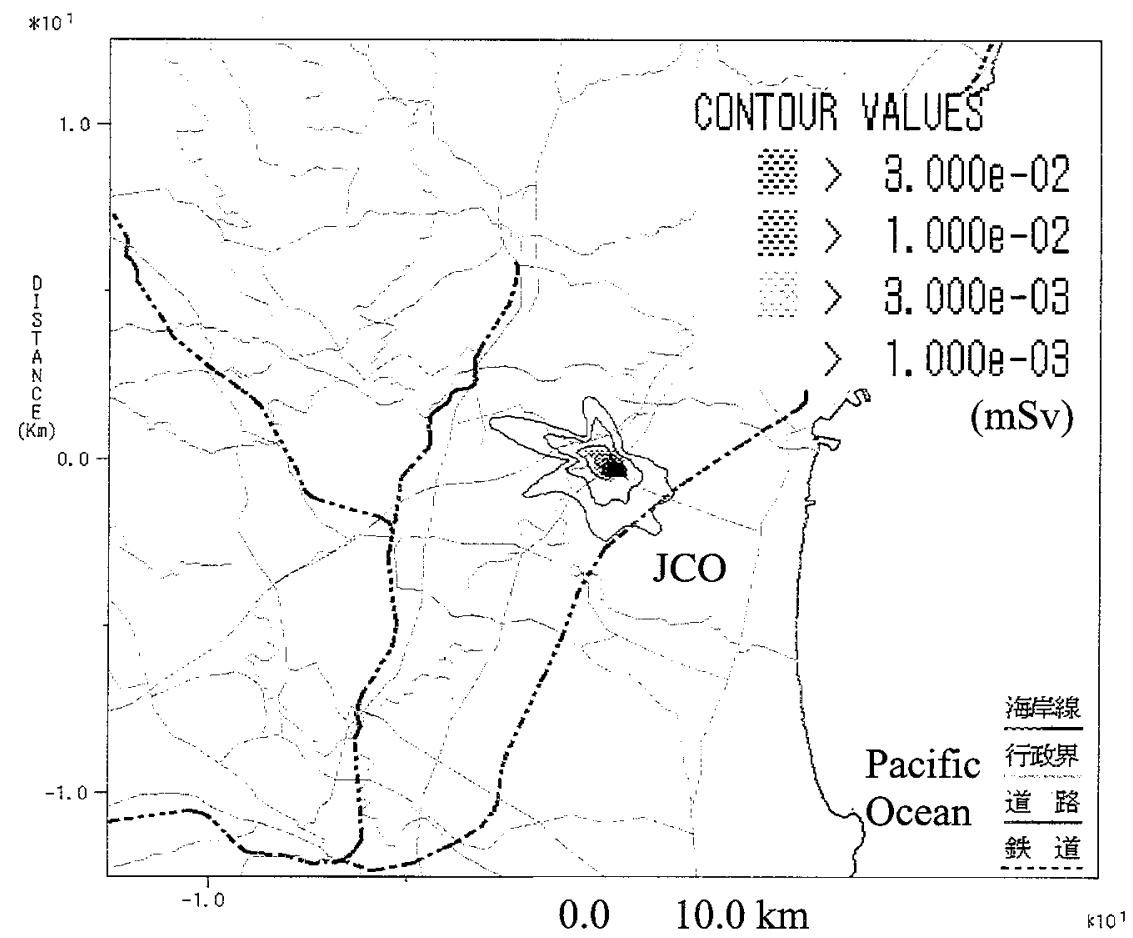

Figure 9. Horizontal distribution of external doses at a height of $1.5 \mathrm{~m}$ above the ground accumulated until 8:00, 1 Oct. (JAERI/WSPEEDI)

During this cooperative activity, the Web proved to be highly effective tool to exchange and compare the prediction results. However, the teleconference tool which uses Internet had a problem due to data traffic, and voice and video were sometimes broken. In the future, the establishment of a link by using a private network, e.g., ISDN, would be desirable in order to maximize the timely exchange of data and results. In the near future InterNet-II may provide bandwidth relief, but we anticipate even this will become saturated in the locale of a real accident. 


\section{CONCLUSIONS}

Under the auspices of a US DOE-JAERI Memorandum of Understanding, JAERI and LLNL agreed to develop and evaluate a prototype information exchange protocol between WSPEEDI and ARAC for nuclear accident emergency situations. The purpose of this project is;

- quick exchange and review of atmospheric modeling products and environmental data during an emergency,

- distribution of predicted results to other countries having no prediction capabilities, and

- utilization of the link for collaborative studies.

The network is constructed on the hardware of the computer platform and the network, Internet. Currently, a Silicon Graphics Indy workstation and Mac PC with a microphone and a video camera serve as the platform at both ends of the connection.

The information exchange tool is the Web site/browser portion and the video-teleconferencing tool. Each Web site permits privileged users to log on and review the computational results for a given facility. Accessible information includes model parameters and assumptions used in the calculations, graphical displays of wind data, and plots of predicted concentrations, dose and deposition.

The video-teleconference has experienced delays of 5-10 seconds. However, unavoidable Internet congestion problems sometimes occurred at the various bridges and gateways between the US and Japan. Similar whiteboard delays were also experienced, but the overall performance of the system was acceptable.

After starting the construction of the link between ARAC and WSPEEDI, we have experienced three cooperative exchanges against accidental releases of radionuclides into the atmosphere, e.g., a bituminization plant for radioactive waste at the former Power Reactor and Nuclear Fuel Development Corporation (PNC) in Japan In 11 March, 1997, an accidental discharge of Cs137 occurred at Algeciras, Spain in 30 May, 1998, and a criticality accident occurred in the conversion building at the nuclear fuel processing facility, JCO Company on 30 September 1999, in Tokai, Japan. These cooperative responses to actual events proved the network would have provided an advantage over the face-to-face exchange, as each participant is acting from their own institutional environments, where all local data and even colleagues are readily accessible. 
JAERI and LLNL intend to extend the network to the European system, RODOS at Forschungszentrum Karlsruhe GmbH (FZK). The first trial for the International Nuclear Emergency Exercise (INEX) sponsored by OECD/NEA experienced the benefits of a direct international data exchange among JAERI, LLNL and FZK. In the near future, the triangular prototype network among the U.S., Europe and Japan will be constructed.

This report and the work performed at LLNL have been supported by the US DOE, National Nuclear Security Administration Office of Defense Nuclear Nonproliferation, International Emergency Cooperation Program

This Work was performed under the auspices of the U.S. Department of Energy by the University of California Lawrence Livermore National Laboratory under Contract No. W-7405-Eng-48.

\section{REFERENCES}

1 [Sullivan, 1993] Sullivan, T. J., Ellis, J. S., Foster, C.S., et al., Atmospheric Release Advisory Capability: Real-Time Modeling of Airborne Hazardous Materials, Bulletin of the American Meteor. Soc., 74, 2343-2361, 1993.

2 [Ellis \& Sullivan 2000] Ellis, J. S. and Sullivan, T.J., An Advanced System for Environmental Emergency Response. Proceedings: International Radiation Protection Association, $10^{\text {th }}$ International Conference, Kyoto, Japan, May 10-14, 2000.

3 [Leone \& Nasstrom \& Maddix, 1997] Leone, J.M., Nasstrom, J.S. and Maddix, D., A First Look at the New ARAC Dispersion Model. Preprint. Amer. Nucl. Svc, $6^{\text {th }}$ Topical Meeting on Emergency Preparedness and Response, San Francisco, April 1997.

4 [Nasstrom, et al., 1999] Nasstrom, J.S., Sugiyama, G., Leone, J.M., and Ermak, D.L., A Real Time Atmospheric Dispersion Modeling System, AMS $11^{\text {th }}$ Joint Conference on Applications of Air Pollution Meteorology, Long Beach, CA. Jan 2000.

5 [Chan \& Sugiyama, 1997] Chan, S. and Sugiyama G., A New Model for Generating MassConsistent Wind Fields Over Continuous Terrain Preprint. Amer. Nucl. Svc, $6^{\text {th }}$ Topical Meeting on Emergency Preparedness and Response, San Francisco, April 1997, 375-378.

6 [Sugiyama \& Chan 1998] Sugiyama, G. and Chan, S. A New Meteorological Data Assimilation Model for Real-Time Emergency Response. Preprint. 10 $10^{\text {th }}$ Joint Conference on the Application of Air Pollution Meteorology. Phoenix, AZ. Jan 11-16, 1998, 285-289. 
7 [Ellis et al., 1997] Ellis, J.S., Lee, R.L., Sumikawa, D.L., and Sullivan, T.J., ARAC and Its Modernisation, Radiation Protection Dosimetry, 73, No. 1-4, pp. 241-245, 1997.

8 [Mirin et al., 1999] Mirin, A.A., Sugiyama, G., Hodur, R.M., Schmidt, J.M., and Chen, S., Design Considerations and Performance of a Scalable Version of a Nonhydrostatic Atmospheric Model. DoD High Performance Computing Modernization Program Users Group Conference, Monterey, CA, June 7-10, 1999, UCRL-JC-133451.

9 [Imai, 1985] Imai, K., Chino, M., Ishikawa, H., et al., SPEEDI: A Computer Code System for the Real-Time Prediction of Radiation Dose to the Public due to an Accidental Release, JAERI1297, Japan Atomic Energy Research Institute, Tokyo, Japan, 1985.

10 [Chino, 1995] Chino, M., Ishikawa, H., Yamazawa, H., et al., WSPEEDI (Worldwide Version of SPEEDI ): Emergency Response System to Predict Radiological Impacts on the Japanese People Due To A Nuclear Accident In Abroad, JAERI-1334, Japan Atomic Energy Research Institute, Tokyo, Japan, 1995.

11 [Nagai, 1999] Nagai, H., Chino, H., Yamazawa, H., Development of Scheme for Predicting Atmospheric Dispersion of Radionuclides during Nuclear Emergency by Using Atmospheric Dynamic Model. J. Nucl. Sci. Techol., 41, 777-785, 1999.

12 [Ishikawa, 1994] Ishikawa, H., Mass-Consistent Wind Models as a Meteorological Preprocessor for Tracer Transport Model. J. Of Applied Meteor., 33, 733-743, 1994.

13 [Ishikawa, 1991] Ishikawa, H., Chino, M., Development of World-wide Version Of System For Prediction Of Environmental Emergency Dose Information: WSPEEDI, (II) Long-range transport model and its application to dispersion of cesium-137 from Chernobyl, J. of Nucl. Sci. Technol. Japan, 28, 642-655, 1991.

14 [Chino, 1997] Chino, M., Nagai, H., Estimated Amount of 137Cs Discharged Into Environment Due To The Accident In The Bituminization Plant At Power Reactor And Nuclear Fuel Development Corporation, J. Nucl. Sci. Technol., 39, 644-646, 1998. (in Japanese)

15 [Yamazawa, 1999] Yamazawa, H., Long-range Dispersion Analysis on Accidental Atmospheric Release of Cesium-137 at Algeciras, J. Nucl. Sci. Technol., 41, 114-116, 1998. (in Japanese) 